\title{
NORMAL FUNCTIONS AND THE HEIGHT OF GROSS-SCHOEN CYCLES
}

\author{
ROBIN DE JONG
}

\begin{abstract}
We prove a variant of a formula due to Zhang relating the BeilinsonBloch height of the Gross-Schoen cycle on a pointed curve with the selfintersection of its relative dualizing sheaf. In our approach, the height of the Gross-Schoen cycle occurs as the degree of a suitable Bloch line bundle. We show that the Chern form of this line bundle is nonnegative, and we calculate its class in the Picard group of the moduli space of pointed stable curves of compact type. The basic tools are normal functions and biextensions associated to the cohomology of the universal Jacobian.
\end{abstract}

\section{$\S 1$. Introduction}

Let $X$ be a smooth geometrically connected curve of genus $g \geq 2$ over a field $k$. Let $e=\sum_{i} a_{i} p_{i}$ be a divisor of degree 1 on $X$. Consider the following cycles of codimension 2 on the triple product $X^{3}$ :

$$
\begin{aligned}
\Delta_{123} & =\{(x, x, x): x \in X\}, \\
\Delta_{12} & =\sum_{i} a_{i}\left\{\left(x, x, p_{i}\right): x \in X\right\}, \\
\Delta_{13} & =\sum_{i} a_{i}\left\{\left(x, p_{i}, x\right): x \in X\right\}, \\
\Delta_{23} & =\sum_{i} a_{i}\left\{\left(p_{i}, x, x\right): x \in X\right\}, \\
\Delta_{1} & =\sum_{i, j} a_{i} a_{j}\left\{\left(x, p_{i}, p_{j}\right): x \in X\right\}, \\
\Delta_{2} & =\sum_{i, j} a_{i} a_{j}\left\{\left(p_{i}, x, p_{j}\right): x \in X\right\},
\end{aligned}
$$

Received April 24, 2012. Revised February 20, 2013. Accepted May 14, 2013.

First published online January 15, 2014.

2010 Mathematics Subject Classification. Primary 14G40; Secondary 14C25, 14D06.

(C) 2014 by The Editorial Board of the Nagoya Mathematical Journal 


$$
\Delta_{3}=\sum_{i, j} a_{i} a_{j}\left\{\left(p_{i}, p_{j}, x\right): x \in X\right\}
$$

and put

$$
\Delta_{e}=\Delta_{123}-\Delta_{12}-\Delta_{13}-\Delta_{23}+\Delta_{1}+\Delta_{2}+\Delta_{3} .
$$

Then $\Delta_{e}$ is a cohomologically trivial cycle on $X^{3}$, studied in detail by Gross and Schoen [13]. Assume that $k$ is either a number field or a function field of a curve, and assume that $X$ has semistable reduction over $k$. Then Gross and Schoen construct in [13] a canonical $\mathbb{R}$-valued height $\left(\Delta_{e}, \Delta_{e}\right)$ associated to $\Delta_{e}$, fitting in a general approach due to Beilinson [4] and Bloch [5]. Let $\mathrm{CH}^{2}\left(X^{3}\right)_{\mathbb{Q}}$ be the Chow group of codimension 2 cycles on $X^{3}$ with rational coefficients. An important feature of the height $\left(\Delta_{e}, \Delta_{e}\right)$ of the Gross-Schoen cycle is that its nonvanishing implies the nontriviality of the class of $\Delta_{e}$ in $\mathrm{CH}^{2}\left(X^{3}\right)_{\mathbb{Q}}$ (see [29, end of Section 1.1]), and hence (according to the Beilinson-Bloch conjecture) the conjectural vanishing of the special value of a suitable $L$-series attached to $X^{3}$. The height of the Gross-Schoen cycle is conjectured to be nonnegative (see [29, Conjecture 1.4.1]).

In a recent paper, Zhang [29] derived an explicit formula for $\left(\Delta_{e}, \Delta_{e}\right)$ and used it to prove some important new results in this direction. We describe the formula, and simply refer the reader to [29] for further discussion of its ramifications.

First of all, for each place $v$ of $k$, Zhang introduces a local $\mathbb{R}$-valued invariant $\varphi\left(X_{v}\right)$ of $X$ at $v$, as follows. Let $\mu_{v}$ be the canonical Arakelov $(1,1)$-form (see [1], [12]) on $X_{v}=X \otimes \bar{k}_{v}$ if $v$ is archimedean, and let $\mu_{v}$ be the canonical admissible metric from [28, Section 3] on the reduction graph $R_{v}$ of $X$ at $v$ if $v$ is non-Archimedean. If $v$ is an archimedean place, let $\Delta_{\mathrm{Ar}}$ be the Laplacian on $L^{2}\left(X_{v}, \mu_{v}\right)$ given by putting $\partial \bar{\partial} f=\pi i \Delta_{\mathrm{Ar}}(f) \mu_{v}$ for $C^{\infty}$-functions $f$. Let $\left(\phi_{\ell}\right)_{\ell=0}^{\infty}$ be an orthonormal basis of real eigenfunctions of $\Delta_{\mathrm{Ar}}$, with corresponding eigenvalues $0=\lambda_{0}<\lambda_{1} \leq \lambda_{2} \leq \cdots$. He then puts

$$
\varphi\left(X_{v}\right)=\sum_{\ell>0} \frac{2}{\lambda_{\ell}} \sum_{m, n=1}^{g}\left|\int_{X_{v}} \phi_{\ell} \omega_{m} \wedge \bar{\omega}_{n}\right|^{2},
$$

where $\left(\omega_{m}\right)_{m=1}^{g}$ is an orthonormal basis for the inner product on $\mathrm{H}^{0}\left(X_{v}, \Omega_{X_{v}}^{1}\right)$ given by putting $(\alpha, \beta)=\frac{i}{2} \int_{X_{v}} \alpha \wedge \bar{\beta}$. If $v$ is a non-Archimedean place, he puts

$$
\varphi\left(X_{v}\right)=-\frac{1}{4} \delta\left(X_{v}\right)+\frac{1}{4} \int_{R_{v}} g_{\mu, v}(x, x)\left((10 g+2) \mu_{v}-\delta_{K_{v}}\right)
$$


where $\delta\left(X_{v}\right)$ is the number of singular points in the geometric special fiber of $X$ at $v, g_{\mu, v}$ is the Green's function (see [28, Section 3]) for the canonical admissible metric $\mu_{v}$ on $R_{v}$, and $K_{v}$ is the canonical divisor on $R_{v}$.

Let $x_{e}$ be the class of the degree 0 divisor $(2 g-2) e-K$ in the Jacobian of $X$, where $K$ is a canonical divisor on $X$, and denote by $\hat{h}\left(x_{e}\right)$ the NéronTate height of $x_{e}$ in $\mathbb{R}$ (we warn the reader that our $x_{e}$ is $2 g-2$ times what is called $x_{e}$ in [29]). Let $(\omega, \omega)_{a}$ be the admissible self-intersection of the relative dualizing sheaf (see [28, Section 4]) of $X$ over $k$. Define local factors $N v$ as follows: if $v$ is a real embedding, then $N v=\exp (1)$; if $v$ is a pair of complex embeddings, then $N v=\exp (2)$; and if $v$ is non-Archimedean, then $N v$ is the cardinality of the residue field at $v$, provided that this residue field is finite, and $N v=\exp (1)$ otherwise. Zhang's formula is then the following.

Theorem 1.1 ([29, Theorem 1.3.1]). The equality

$$
\left(\Delta_{e}, \Delta_{e}\right)=\frac{2 g+1}{2 g-2}(\omega, \omega)_{a}+\frac{3}{2 g-2} \hat{h}\left(x_{e}\right)-\sum_{v} \varphi\left(X_{v}\right) \log N v
$$

holds in $\mathbb{R}$.

The purpose of this article is to prove a variant of Zhang's formula. The main aspect of our approach is that the archimedean contributions $\varphi\left(X_{v}\right)$ from Zhang's formula appear as a norm at infinity of a certain canonical isomorphism of line bundles related to $\Delta_{e}, \omega$, and $x_{e}$.

Let $S$ be a smooth quasiprojective variety over the complex numbers. Let $\pi: \mathcal{X} \rightarrow S$ be a smooth projective family of curves of genus $g \geq 2$ over $S$, and assume that a flat divisor $e$ of relative degree 1 on $\mathcal{X} / S$ is given. Let $\omega$ be the relative dualizing sheaf of $\mathcal{X} / S$, and let $x_{e}$ be the divisor class of relative degree 0 on $\mathcal{X} / S$ given by $(2 g-2) e-c_{1}(\omega)$. Let $\langle\omega, \omega\rangle$ on $S$ be the Deligne pairing (see [10]) of $\omega$ with itself, and let $\left\langle x_{e}, x_{e}\right\rangle$ on $S$ be the Deligne pairing of $x_{e}$ with itself. Let $\Delta_{e}$ be the flat relative Gross-Schoen cycle with base point $e$ on the triple self-fiber product of $\mathcal{X} / S$.

According to Bloch [6] (see also Section 2 below), we have a canonical algebraic line bundle $\left\langle\Delta_{e}, \Delta_{e}\right\rangle$ associated to $\Delta_{e}$ on $S$. The formation of Bloch's line bundle is compatible with base change, and if $S$ is a smooth projective connected curve, then $\operatorname{deg}_{S}\left\langle\Delta_{e}, \Delta_{e}\right\rangle$ equals the height $\left(\Delta_{e}, \Delta_{e}\right)$ of the Gross-Schoen cycle on the generic fiber of $\mathcal{X} / S$.

Each of the algebraic line bundles $\left\langle\Delta_{e}, \Delta_{e}\right\rangle,\langle\omega, \omega\rangle$, and $\left\langle x_{e}, x_{e}\right\rangle$ is equipped with a canonical Hermitian metric. For both Deligne pairings this is in [10], and for the Bloch pairing $\left\langle\Delta_{e}, \Delta_{e}\right\rangle$ we refer to Sections 2 and 3 below. Note 
that the archimedean $\varphi$-invariants of the fibers of $\mathcal{X} \rightarrow S$ define a real-valued function on $S$. In Section 7 we show that the function $\varphi$ is continuous for the complex topology on $S$.

The following theorem is our variant of Zhang's result.

THEOREM 1.2. There exists an isomorphism of algebraic line bundles

$$
\left\langle\Delta_{e}, \Delta_{e}\right\rangle^{\otimes 2 g-2} \stackrel{\sim}{\rightarrow}\langle\omega, \omega\rangle^{\otimes 2 g+1} \otimes\left\langle x_{e}, x_{e}\right\rangle^{\otimes-3}
$$

on $S$, canonical up to a sign, and having norm $\exp (-(2 g-2) \varphi)$.

To prove Theorem 1.2, we reduce to the universal case where $S$ is the orbifold $\mathcal{P i c}_{g}^{1}$ of pairs $(X, e)$ consisting of a complex smooth projective connected curve $X$ of genus $g$ together with a divisor class $e$ of degree 1 on $X$. We conjecture that an analogue of Theorem 1.2 holds for smooth quasiprojective varieties $S$ over complete discretely valued fields, featuring the non-Archimedean $\varphi$-invariant introduced above. In combination with Theorem 1.2, such an analogue would yield Zhang's theorem as an immediate corollary.

We prove Theorem 1.2 by considering normal functions and biextensions on a fibration of intermediate Jacobians over $\mathcal{P i c}_{g}^{1}$. As such, our approach is rather different from the one pursued in Zhang's original paper [29]. We think that as a result of our approach the Hodge theoretic and motivic aspects of Zhang's article become more visible. For example, the motive $M$ appearing in [29, Section 5] corresponds to the intermediate Jacobian $\mathcal{J}\left(\wedge^{3} H / H\right)$ studied in Section 4 below.

The Hermitian line bundle $\left\langle\Delta_{e}, \Delta_{e}\right\rangle$ on $\mathcal{P i c}_{g}^{1}$ has a unique extension as a Hermitian line bundle over $\mathcal{P i c}_{g}^{1, c}$, the universal Picard variety of degree 1 over the moduli space $\mathcal{M}_{g}^{c}$ of stable curves of compact type. We denote this extension over $\mathcal{P i c}_{g}^{1, c}$ by the same symbol.

THEOREM 1.3. The line bundle $\left\langle\Delta_{e}, \Delta_{e}\right\rangle$ has nonnegative Chern form and hence is nef on $\mathcal{P i c}_{g}^{1, c}$.

As a corollary of Theorem 1.3, we obtain the nonnegativity of the height of the Gross-Schoen cycle for curves over function fields in characteristic zero with everywhere stable reduction of compact type. We note that this also follows from [30] by an application of the Hodge index theorem.

To finish, we compute the first Chern class of the Bloch line bundle $\left\langle\Delta_{e}, \Delta_{e}\right\rangle$ restricted to the universal 1-pointed stable curve of compact type 
$\mathcal{M}_{g, 1}^{c}$. Let $\psi$ be the first Chern class of the pullback, along the canonical section, of the relative dualizing sheaf of the universal curve over $\mathcal{M}_{g, 1}^{c}$, and let $\lambda$ be the first Chern class of the Hodge bundle. For $1 \leq i \leq g-1$ an integer, let $\Delta_{i}^{\{x\}}$ be the boundary divisor in $\mathcal{M}_{g, 1}^{c}$ of which the generic point is a reducible curve with precisely one node and two smooth irreducible components, one of genus $i$ and the other of genus $g-i$, such that the marked point lies on the genus $i$ component minus the node. Let $\delta_{i}^{\{x\}}$ be the class of $\Delta_{i}^{\{x\}}$ in the Picard group of $\mathcal{M}_{g, 1}^{c}$. According to [2, Theorem 2], the group $\operatorname{Pic}\left(\mathcal{M}_{g, 1}^{c}\right)$ is generated by the classes $\psi, \lambda$, and $\delta_{i}^{\{x\}}$ (and freely so if $g \geq 3$ ).

TheOREM 1.4. Assume that $g \geq 3$. Then we have the equality

$$
\left\langle\Delta_{e}, \Delta_{e}\right\rangle=6 g \psi+12 \lambda-\sum_{i=1}^{g-1} 6 i \delta_{g-i}^{\{x\}}
$$

in $\operatorname{Pic}\left(\mathcal{M}_{g, 1}^{c}\right)$

Observe that the class of $\left\langle\Delta_{e}, \Delta_{e}\right\rangle$ in the Picard group of $\mathcal{M}_{g, 1}^{c}$ is divisible by 6 . It would be interesting to have a description of a natural sixth root of $\left\langle\Delta_{e}, \Delta_{e}\right\rangle$.

This article is organized in the following form. We discuss generalities on Bloch's pairing, biextensions, and variations of polarized Hodge structure in Sections 2, 3, and 4, respectively. In Sections 5 and 6, we study normal functions associated to the Ceresa cycle in the Jacobian of a curve. The connection with the Gross-Schoen cycle is made in Section 7, which also contains a proof of Theorem 1.2. Finally, Theorems 1.3 and 1.4 are proved in Section 8.

\section{$\S 2$. Bloch's pairing between cycle classes}

We begin by reviewing the construction, due to Bloch [6], of a canonical algebraic line bundle $\langle z, w\rangle$ associated to a pair of relatively homologically trivial algebraic cycle classes $z, w$ on a family of smooth complex projective varieties. In Section 3, we discuss an alternative formulation of the same pairing in terms of biextensions and normal functions on the associated family of Griffiths intermediate Jacobians. For a more extensive discussion of these topics, proofs, and broader generality, we refer the reader to the sources [6], [21], [24], and [26]. 
Let $S$ be a smooth quasiprojective variety over $\mathbb{C}$, endowed with the analytic topology. We consider a smooth projective morphism $\pi: \mathcal{X} \rightarrow S$ of relative dimension $d$ over $S$. For each integer $1 \leq p \leq d$, we let $\mathrm{CH}_{o}^{p}(\mathcal{X} / S)$ denote the sheaf of abelian groups on $S$ associated to the presheaf which associates to each open subset $U$ of $S$ the group of codimension $p$ algebraic cycles on $\mathcal{X}$ that restrict to a homologically trivial cycle in each of the geometric fibers of $\mathcal{X} / S$, modulo rational equivalence.

Let $p, q$ be positive integers such that $p+q=d+1$. Let $z, w$ be global sections of $\mathrm{CH}_{o}^{p}(\mathcal{X} / S)$ and $\mathrm{CH}_{o}^{q}(\mathcal{X} / S)$, respectively. In [6] a canonical line bundle $\langle z, w\rangle$ is constructed on $S$. The construction is bi-additive in $z, w$, and can be summarized as follows. For each open subset $U$ of $S$, locally over $U$ the line bundle $\langle z, w\rangle$ is generated by symbols $\langle Z, W\rangle$, where $Z, W$ are algebraic cycles representing $z, w$ over $U$, with disjoint support, such that the following relations hold.

Let $\langle Z, W\rangle$ and $\left\langle Z, W^{\prime}\right\rangle$ be local symbols over $U$, and suppose that $W, W^{\prime}$ are rationally equivalent over $U$. This essentially means that there exists a cycle $Y$ on $\mathcal{X}_{U}$ and a rational function $f$ on $Y$ such that $W-W^{\prime}=\operatorname{div}_{Y}(f)$; we assume that we can choose $Y$ such that $Y$ and $Z$ intersect properly. Consider then the intersection cycle $Y . Z$; we assume that the support of $Y . Z$ is finite flat over $S$. Note that as $Z, W$ and $Z, W^{\prime}$ have disjoint support the rational function $f$ restricts to an invertible regular function on $Y . Z$. Let $\sigma_{Z}\left(W-W^{\prime}\right)$ in $\mathcal{O}_{S}^{*}(U)$ be the result of applying the norm map from $Y . Z$ to $S$ to the restriction of $f$ to $Y . Z$; then we put $\langle Z, W\rangle=\sigma_{Z}\left(W-W^{\prime}\right) \cdot\left\langle Z, W^{\prime}\right\rangle$.

Likewise, if $\langle Z, W\rangle$ and $\left\langle Z^{\prime}, W\right\rangle$ are local symbols over $U$ with $Z, Z^{\prime}$ rationally equivalent over $U$, with a cycle $Y^{\prime}$ on $\mathcal{X}_{U}$ intersecting $W$ properly and a rational function $g$ on $Y^{\prime}$ such that $Z-Z^{\prime}=\operatorname{div}_{Y^{\prime}}(g)$, we put $\langle Z, W\rangle=\sigma_{W}\left(Z-Z^{\prime}\right) \cdot\left\langle Z^{\prime}, W\right\rangle$, where $\sigma_{W}\left(Z-Z^{\prime}\right)$ in $\mathcal{O}_{S}^{*}(U)$ is the result of applying the norm map from $Y^{\prime} . W$ to $S$ to the invertible regular function obtained by restricting $g$ to $Y^{\prime} . W$.

We mention the cocycle relations

$$
\sigma_{Z}\left(W-W^{\prime}\right) \cdot \sigma_{W^{\prime}}\left(Z-Z^{\prime}\right)=\sigma_{W}\left(Z-Z^{\prime}\right) \cdot \sigma_{Z^{\prime}}\left(W-W^{\prime}\right)
$$

in $\mathcal{O}_{S}^{*}(U)$ for $Z, W, Z^{\prime}, W^{\prime}$ as above.

In [6], a torsor $\mathbb{E}$ is constructed over the product $\mathrm{CH}_{o}^{p}(\mathcal{X} / S) \times \mathrm{CH}_{o}^{q}(\mathcal{X} / S)$. The above $\langle z, w\rangle$ are the fibers of this torsor. The various properties of the torsor $\mathbb{E}$ discussed in [6] readily yield the following proposition. 
Proposition 2.1. We have the following.

(a) (Symmetry) Let $z, w$ be global sections of $\mathrm{CH}_{o}^{p}(\mathcal{X} / S)$ and $\mathrm{CH}_{o}^{q}(\mathcal{X} / S)$, respectively. Then there exists a canonical isomorphism $\langle z, w\rangle \stackrel{\sim}{\rightarrow}\langle w, z\rangle$ of line bundles on $S$.

(b) (Biadditivity) Let $z, z^{\prime}$ be global sections of $\mathrm{CH}_{o}^{p}(\mathcal{X} / S)$, and let $w, w^{\prime}$ be global sections of $\mathrm{CH}_{o}^{q}(\mathcal{X} / S)$. Then there are canonical isomorphisms

$$
\left\langle z+z^{\prime}, w\right\rangle \stackrel{\sim}{\rightarrow}\langle z, w\rangle \otimes\left\langle z^{\prime}, w\right\rangle,\left\langle z, w+w^{\prime}\right\rangle \stackrel{\sim}{\rightarrow}\langle z, w\rangle \otimes\left\langle z, w^{\prime}\right\rangle
$$

of line bundles on $S$.

(c) (Projection formula) Let $\mathcal{X} / S$ and $\mathcal{Y} / S$ be smooth projective families with $\mathcal{X} / S$ of relative dimension $d$. Let $f: \mathcal{X} \rightarrow \mathcal{Y}$ be a proper flat $S$ morphism. Let $z$ be a global section of $\mathrm{CH}_{o}^{p}(\mathcal{X} / S)$, and let $w$ be a global section of $\mathrm{CH}_{o}^{d+1-p}(\mathcal{Y} / S)$. Then there exists a canonical isomorphism $\left\langle f_{*} z, w\right\rangle \stackrel{\sim}{\rightarrow}\left\langle z, f^{*} w\right\rangle$ of line bundles on $S$.

Proof. The symmetry derives from [6, Proposition 4], and the bi-additivity boils down to the biextension property of the torsor $\mathbb{E}$ verified in [6, Proposition 7]. The projection formula follows from [26, Satz 7.10].

Bloch's pairing is to be considered as an intersection pairing. In fact, let $z, w$ be global sections of $\mathrm{CH}_{o}^{p}(\mathcal{X} / S)$ and $\mathrm{CH}_{o}^{q}(\mathcal{X} / S)$, respectively, where $p+q=d+1$. Let $Z, W$ be global representatives of $z, w$ intersecting properly on $\mathcal{X}$, and let $Z . W$ be their intersection cycle. Then there is a noncanonical isomorphism of line bundles $\langle z, w\rangle \stackrel{\sim}{\rightarrow} \mathcal{O}_{S}\left(\pi_{*}(Z . W)\right.$ ) on $S$ (see [26, Satz 6.8]). In particular, if $S$ is a smooth projective curve, one has $\operatorname{deg}_{S}\langle z, w\rangle=$ $\operatorname{deg}(Z . W)$.

Let $S$ again be an arbitrary smooth complex quasiprojective variety, and consider cycle classes $z, w$ as above. Then the line bundle $\langle z, w\rangle$ carries a canonical Hermitian metric $\|\cdot\|$. It is determined as follows (see [21, Section II.4]). Let $\langle Z, W\rangle$ be a local generating section of $\langle z, w\rangle$ over the open subset $U$ of $S$. In particular, the cycles $Z, W$ have disjoint support over $U$. There exists a Green's current $g_{W}$ for the cycle $W$ on $\mathcal{X}_{U}$ such that $\partial \bar{\partial} g_{W}+\pi i \delta_{W}$ is a $(q, q)$-form on $\mathcal{X}_{U}$, and such that $g_{W}$ vanishes in each fiber of $\mathcal{X}_{U} / U$. Write $\langle Z, W\rangle_{\infty}=-\log \|\langle Z, W\rangle\|$. Then $\langle Z, W\rangle_{\infty}$ is given by the identity

$$
\langle Z, W\rangle_{\infty}=-\int_{\pi} \delta_{Z} \wedge g_{W}
$$

that is, the archimedean height pairing of $Z, W$. It can be verified using Stokes's theorem that $\langle Z, W\rangle_{\infty}$ is independent of the choice of $g_{W}$, and is symmetric in $Z, W$. In fact, we have the following result due to Meyer. 
Proposition 2.2 ([21, Satz 4.3.2]). Each of the canonical isomorphisms of Proposition 2.1 is an isometry if Bloch's pairing is endowed with the canonical Hermitian metric determined by the archimedean height pairing.

\section{§3. Poincaré biextensions}

An alternative approach to Bloch's line bundle valued pairing uses Poincaré biextensions on intermediate Jacobians. References for the results in this section are [14], [21], and [24]. Let $\pi: \mathcal{X} \rightarrow S$ again be a smooth projective morphism of relative dimension $d$, with $S$ a smooth complex quasiprojective variety, and consider again two positive integers $p, q$ with $p+q=d+1$. We denote by $\mathcal{J}^{p}(\mathcal{X} / S)$ and $\mathcal{J}^{q}(\mathcal{X} / S)$ the Griffiths intermediate Jacobian fibrations associated to the variations of Hodge structure $\mathrm{R}^{2 p-1} \pi_{*} \mathbb{Z}_{\mathcal{X}}$ and $\mathrm{R}^{2 q-1} \pi_{*} \mathbb{Z}_{\mathcal{X}}$ on $S$. Let $\check{\mathcal{J}}^{p}(\mathcal{X} / S)$ be the dual torus fibration of $\mathcal{J}^{p}(\mathcal{X} / S)$. Then there exists a canonical isomorphism

$$
\operatorname{pd}: \mathcal{J}^{q}(\mathcal{X} / S) \stackrel{\sim}{\rightarrow} \check{\mathcal{J}}^{p}(\mathcal{X} / S)
$$

of torus fibrations over $S$, induced by Poincaré duality.

Let $\mathcal{B}$ be the canonical Poincaré line bundle over $\mathcal{J}^{p}(\mathcal{X} / S) \times \check{\mathcal{J}}^{p}(\mathcal{X} / S)$ (see [14, Section 3.2]). Here and henceforth, products are fiber products over the base variety $S$. The Poincaré bundle $\mathcal{B}$ is holomorphic, and comes with a trivialization $e^{*} \mathcal{B} \stackrel{\sim}{\rightarrow} \mathcal{O}_{S}$ along the zero section $e$ of $\mathcal{J}^{p}(\mathcal{X} / S) \times \check{\mathcal{J}}^{p}(\mathcal{X} / S)$ over $S$. For local sections $z, w$ of $\mathrm{CH}_{o}^{p}(\mathcal{X} / S)$ and $\mathrm{CH}_{o}^{q}(\mathcal{X} / S)$, represented by algebraic cycles, we denote by $a_{p}(z), a_{q}(w)$ their respective fiberwise Griffiths Abel-Jacobi images in $\mathcal{J}^{p}(\mathcal{X} / S)$ and $\mathcal{J}^{q}(\mathcal{X} / S)$. Following terminology due to P. Griffiths, the pair $\left(a_{p}(z), \operatorname{pd} a_{q}(w)\right)$ is a local normal function section from $S$ into $\mathcal{J}^{p}(\mathcal{X} / S) \times \check{\mathcal{J}}^{p}(\mathcal{X} / S)$, and is in particular holomorphic.

The connection with Bloch's pairing is expressed by the next proposition.

Proposition 3.1. Let $z, w$ be global sections of $\mathrm{CH}_{o}^{p}(\mathcal{X} / S)$ and $\mathrm{CH}_{o}^{q}(\mathcal{X} / S)$, respectively. Then there exists a canonical isomorphism of holomorphic line bundles

$$
\langle z, w\rangle \stackrel{\sim}{\rightarrow}\left(a_{p}(z), \operatorname{pd} a_{q}(w)\right)^{*} \mathcal{B}
$$

on $S$.

Proof. Let $\langle Z, W\rangle$ be a local trivializing section of $\langle z, w\rangle$ over an open subset $U \subset S$. In [14, Section 3.3] a construction is given of a holomorphic section $b_{Z, W}$ of $\left(a_{p}(z), \operatorname{pd} a_{q}(w)\right)^{*} \mathcal{B}$ over $U$, natural in $Z, W$. These local constructions glue together into a morphism $\langle z, w\rangle \stackrel{\sim}{\rightarrow}\left(a_{p}(z), \operatorname{pd} a_{q}(w)\right)^{*} \mathcal{B}$ 
of holomorphic line bundles over $S$. By [24, Theorem 1], this morphism is an isomorphism in each fiber. The proposition follows.

Corollary 3.2. Let $z, w$ be global sections of $\mathrm{CH}_{o}^{p}(\mathcal{X} / S)$ and $\mathrm{CH}_{o}^{q}(\mathcal{X} / S)$, respectively, represented by flat families of cycles $Z, W$. Then the formation of the line bundle $\langle z, w\rangle$ commutes with arbitrary base change. That is, let $f: T \rightarrow S$ with $T$ smooth be a morphism of quasiprojective complex varieties, and let $\mathcal{X}^{\prime}=\mathcal{X} \times{ }_{S} T \rightarrow T$ be the pullback of $\pi: \mathcal{X} \rightarrow S$ along $f: T \rightarrow S$. By pullback we obtain global sections $f^{*} z, f^{*} w$ of $\mathrm{CH}_{o}^{p}\left(\mathcal{X}^{\prime} / T\right)$ and $\mathrm{CH}_{o}^{q}\left(\mathcal{X}^{\prime} / T\right)$, respectively, represented by flat families of cycles over $T$. There exists a canonical isomorphism

$$
f^{*}\langle z, w\rangle \stackrel{\sim}{\longrightarrow}\left\langle f^{*} z, f^{*} w\right\rangle
$$

of line bundles on $T$.

Proof. By the topological proper base change theorem (see [19, Section III.6]), as $\pi$ is proper, and $S, T$ are locally compact for the complex topology, the formation of $\mathrm{R}^{i} \pi_{*} \mathbb{Z}_{\mathcal{X}}$ is compatible with the base change $T \rightarrow S$, for each $i \in \mathbb{Z}$. By functoriality, it follows that the formation of the families of intermediate Jacobians $\mathcal{J}^{i}(\mathcal{X} / S)$ for all $i \in \mathbb{Z}$, the Poincaré biextensions $\mathcal{B}$, and the Poincaré duality isomorphisms pd are compatible with the base change $T \rightarrow S$. For the fiberwise Griffiths Abel-Jacobi images $a_{p}(w), a_{q}(z)$ we further have that

$$
\left(a_{p}\left(f^{*} z\right), \operatorname{pd} a_{q}\left(f^{*} w\right)\right)=f^{*}\left(a_{p}(z), \operatorname{pd} a_{q}(w)\right)
$$

The corollary now follows by applying Proposition 3.1.

According to [14, Section 3.2], the biextension line bundle $\mathcal{B}$ is endowed with a canonical Hermitian metric. This Hermitian metric has the following two properties: (i) its Chern form is translation-invariant in each of the fibers of $\mathcal{J}^{p}(\mathcal{X} / S) \times \check{\mathcal{J}}^{p}(\mathcal{X} / S)$ over $S$; (ii) the trivialization $e^{*} \mathcal{B} \stackrel{\sim}{\rightarrow} \mathcal{O}_{S}$ is an isometry, where $\mathcal{O}_{S}$ is endowed with the trivial metric.

An important result is then the following.

Proposition 3.3. For $\mathcal{B}$ endowed with its canonical Hermitian metric, and for Bloch's pairing $\langle z, w\rangle$ endowed with the canonical Hermitian metric determined by the archimedean height pairing (2.1), the canonical isomorphism from Proposition 3.1 is an isometry. 
For a proof, see [21, Section II.4].

A key example is the case where each of $p, q$ and $d$ is equal to one, that is, where $\mathcal{X} / S$ is a smooth projective morphism of relative dimension one, and $z, w$ are represented by algebraic cycles on $\mathcal{X}$ of relative dimension and degree 0 . In this case, $\mathcal{J}^{p}(\mathcal{X} / S)$ is the Jacobian fibration $\mathcal{J}(\mathcal{X} / S)$ of $\mathcal{X} / S$, and we have a canonical principal polarization $i: \mathcal{J}^{p}(\mathcal{X} / S) \stackrel{\sim}{\rightarrow} \check{\mathcal{J}}^{p}(\mathcal{X} / S)$ as in [22, Définition 2.6.4]. It can be derived from [22, Section 2.7.9] that we have $\mathrm{pd}=i \circ[-1]$.

Assume that there exists a relatively ample line bundle $\mathcal{L}$ on $\mathcal{J}(\mathcal{X} / S)$ over $S$ such that $\pi_{*} \mathcal{L}$ is a line bundle on $S$. Identify $\mathcal{J}(\mathcal{X} / S)$ with its dual using $i$. Then the Poincaré bundle on $\mathcal{J}(\mathcal{X} / S) \times \mathcal{J}(\mathcal{X} / S)$ can be explicitly written as

$$
\mathcal{B}=m^{*} \mathcal{L} \otimes p_{1}^{*} \mathcal{L}^{\otimes-1} \otimes p_{2}^{*} \mathcal{L}^{\otimes-1} \otimes e^{*} \mathcal{L}
$$

where $m: \mathcal{J}(\mathcal{X} / S) \times \mathcal{J}(\mathcal{X} / S) \rightarrow \mathcal{J}(\mathcal{X} / S)$ is the addition map and where $p_{1}, p_{2}: \mathcal{J}(\mathcal{X} / S) \times \mathcal{J}(\mathcal{X} / S) \rightarrow \mathcal{J}(\mathcal{X} / S)$ are the projections on the first and second factor, respectively. The Hermitian line bundle $\langle z, w\rangle$ on $S$ coincides with Deligne's pairing in [10] of $z$ and $w$, and we have canonical isometries

$$
\langle z, w\rangle \stackrel{\sim}{\rightarrow}\left(a_{1}(z),-a_{1}(w)\right)^{*} \mathcal{B} \stackrel{\sim}{\rightarrow}\left(a_{1}(z), a_{1}(w)\right)^{*} \mathcal{B}^{\otimes-1} .
$$

If $S$ is a smooth projective curve, the degree of the line bundle $\left(a_{1}(z)\right.$, $\left.a_{1}(w)\right)^{*} \mathcal{B}$ on $S$ coincides with the Néron-Tate height pairing between the restrictions of the divisor classes $z, w$ to the generic fiber of $\mathcal{X} / S$.

\section{§4. Variations of polarized Hodge structure over $\mathcal{A}_{g}$}

The constructions related to biextensions from Section 3 can be carried out in a somewhat more general setting, namely, general variations of (polarized) Hodge structure. We consider the case where the base space is the moduli space $\mathcal{A}_{g}$ of complex principally polarized abelian varieties of dimension $g$. We view $\mathcal{A}_{g}$ as an orbifold, and assume that $g \geq 2$ throughout. The basic references for this section are [14] and [17].

The starting point of our discussion is a polarized integral Hodge structure $\left(V_{\mathbb{Z}}, Q: \wedge^{2} V_{\mathbb{Z}} \rightarrow \mathbb{Z}(-1)\right)$ of weight -1 , together with a representation $\mathrm{Sp}_{2 g} \rightarrow \operatorname{Sp}\left(V_{\mathbb{Z}}, Q\right)$. By a standard construction (see [27, Theorem 2.5.15]), noting that $\mathrm{Sp}_{2 g}$ is the orbifold fundamental group of $\mathcal{A}_{g}$, these data determine a variation of polarized Hodge structures $\left(\mathcal{V}_{\mathbb{Z}}, \mathcal{Q}\right)$ over $\mathcal{A}_{g}$ such that the fibers of $\mathcal{V}_{\mathbb{Z}}$ are equal to $V_{\mathbb{Z}}$. 
We denote by $\mathcal{J}\left(V_{\mathbb{Z}}\right)$ the Griffiths intermediate Jacobian fibration over $\mathcal{A}_{g}$ associated to $\mathcal{V}_{\mathbb{Z}}$. By definition, if $V_{A}$ is the fiber of the local system $\mathcal{V}_{\mathbb{Z}}$ at the point $A$ of $\mathcal{A}_{g}$, the fiber of $\mathcal{J}\left(V_{\mathbb{Z}}\right)$ at $A$ is the complex torus $J\left(V_{A}\right)=$ $\left(V_{A} \otimes \mathbb{C}\right) /\left(F^{0}\left(V_{A} \otimes \mathbb{C}\right)+\operatorname{Im} V_{A}\right)$. This torus can be canonically identified with the extension group $\operatorname{Ext}^{1}\left(\mathbb{Z}, V_{A}\right)$ in the category of mixed Hodge structures.

The holomorphic tangent bundle of $J\left(V_{A}\right)$ comes equipped with a canonical Hermitian inner product derived from $Q$. This Hermitian inner product determines a translation-invariant differential $(1,1)$-form on $J\left(V_{A}\right)$. It extends over $\mathcal{J}\left(V_{\mathbb{Z}}\right)$ in a canonical way.

Proposition-Definition 4.1. There exists a unique $(1,1)$-form $w_{V}$ on the torus fibration $\mathcal{J}\left(V_{\mathbb{Z}}\right)$ such that (i) its restriction to each fiber over $\mathcal{A}_{g}$ is the translation-invariant $(1,1)$-form associated to $Q$, and (ii) its pullback along the zero section vanishes.

Proof. See [17, Section 5].

Let $\check{\mathcal{J}}\left(V_{\mathbb{Z}}\right)$ be the complex torus fibration dual to $\mathcal{J}\left(V_{\mathbb{Z}}\right)$. Let $\mathcal{B}$ be the canonical Poincaré biextension line bundle on $\mathcal{J}\left(V_{\mathbb{Z}}\right) \times \check{J}\left(V_{\mathbb{Z}}\right)$ (see $[14$, Section 3.2]). Here and henceforth, products are taken over the moduli space $\mathcal{A}_{g}$. Based on what we saw in Section 3, the Poincaré biextension line bundle is a holomorphic line bundle on $\mathcal{J}\left(V_{\mathbb{Z}}\right) \times \check{\mathcal{J}}\left(V_{\mathbb{Z}}\right)$, equipped with a trivialization along the zero section, and a canonical Hermitian metric.

The given polarization $Q$ of $V_{\mathbb{Z}}$ induces an isogeny $i_{Q}: \mathcal{J}\left(V_{\mathbb{Z}}\right) \rightarrow \check{\mathcal{J}}\left(V_{\mathbb{Z}}\right)$ of torus fibrations. We define $\hat{\mathcal{B}}$ to be the pullback, along $\left(\mathrm{id}, i_{Q}\right)$, of the line bundle $\mathcal{B}$ to $\mathcal{J}\left(V_{\mathbb{Z}}\right)$. The bundle $\hat{\mathcal{B}}$ comes endowed with a trivialization along the zero section and canonical Hermitian metric, both induced from $\mathcal{B}$ by pulling back. By abuse of language, we refer to the Hermitian line bundle $\hat{\mathcal{B}}$ as the biextension line bundle over $\mathcal{J}\left(V_{\mathbb{Z}}\right)$.

Proposition 4.2. Let $\mathcal{V}_{\mathbb{Z}}$ be a variation of polarized Hodge structure of weight -1 over $\mathcal{A}_{g}$, and let $\hat{\mathcal{B}}$ be the associated biextension line bundle over $\mathcal{J}\left(V_{\mathbb{Z}}\right)$. Then the canonical Hermitian metric on $\hat{\mathcal{B}}$ satisfies the following two properties: (i) its Chern form equals $2 w_{V}$, and (ii) the pullback of $\hat{\mathcal{B}}$ along the zero section is, via its given trivialization, equal to the trivial Hermitian line bundle. The bundle $\hat{\mathcal{B}}$ together with its canonical metric is uniquely characterized, as a holomorphic Hermitian line bundle on $\mathcal{J}\left(V_{\mathbb{Z}}\right)$, by these two properties. 
Proof. We refer to [17, Proposition 7.3] for the proof that properties (i) and (ii) hold for the canonical metric on $\hat{\mathcal{B}}$. The uniqueness of $\hat{\mathcal{B}}$ as a holomorphic Hermitian line bundle on $\mathcal{J}\left(V_{\mathbb{Z}}\right)$ satisfying (i) and (ii) is stated in [17, Proposition 6.1].

We note that the constructions of Section 3 fit in the present discussion, if one starts out with the universal abelian scheme $\pi: \mathcal{X}_{g} \rightarrow \mathcal{A}_{g}$ and takes the local systems $\mathcal{V}_{\mathbb{Z}, 2 p-1}=\mathrm{R}^{2 p-1} \pi_{*} \mathbb{Z}_{\mathcal{X}_{g}}$ over $\mathcal{A}_{g}$ for positive integers $p$. Then $\mathcal{J}^{p}\left(\mathcal{X}_{g} / \mathcal{A}_{g}\right)=\mathcal{J}\left(V_{\mathbb{Z}, 2 p-1}\right)$ for each positive integer $p$, and for positive integers $p, q$ with $p+q=g+1$ one has a canonical isomorphism pd: $\mathcal{J}\left(V_{\mathbb{Z}, 2 q-1}\right) \stackrel{\sim}{\rightarrow}$ $\check{\mathcal{J}}\left(V_{\mathbb{Z}, 2 p-1}\right)$ of torus fibrations over $\mathcal{A}_{g}$ induced by Poincaré duality.

A fundamental case is the case where $\left(V_{\mathbb{Z}}, Q\right)$ equals $\left(H, Q_{H}\right)$, where $H=H_{1}(X, \mathbb{Z})$ is the first homology group of a compact Riemann surface of genus $g \geq 2$, and $Q_{H}$ is its standard intersection form. By standard Hodge theory of complex tori, one has canonical isomorphisms $\mathcal{V}_{\mathbb{Z}, k} \stackrel{\sim}{\rightarrow} \wedge^{k} \mathcal{V}_{\mathbb{Z}}$, and the polarizations on the $\mathcal{V}_{\mathbb{Z}, k}$ are compatible with these isomorphisms.

An important role in our discussion will be played by the case $k=3$. Note that the Hodge structure $H$ is mapped into $\wedge^{3} H$ by sending $x$ to $x \wedge \zeta$, where $\zeta$ in $\wedge^{2} H$ is the dual of $Q_{H}$. The polarization $Q_{\wedge^{3} H}$ on the Hodge structure $\wedge^{3} H$ sends $\left(x_{1} \wedge x_{2} \wedge x_{3}, y_{1} \wedge y_{2} \wedge y_{3}\right)$ to $\operatorname{det}\left(x_{i}, y_{j}\right)$.

Next consider the Hodge structure $\wedge^{3} H / H$ given as the cokernel of the map $-\wedge \zeta: H \rightarrow \wedge^{3} H$. It carries a polarization given as follows. First of all one has a contraction map $c: \wedge^{3} H \rightarrow H$, defined by

$$
x \wedge y \wedge z \mapsto(x, y) z+(y, z) x+(z, x) y
$$

The composite $H \rightarrow \wedge^{3} H \rightarrow H$ induced by $c$ and $\wedge \zeta$ equals $(g-1)$ times the identity. Denote the projection $\wedge^{3} H \rightarrow \wedge^{3} H / H$ by $p$. After tensoring with $\mathbb{Q}$, the projection $p$ allows a splitting $j$, defined by

$$
p(x \wedge y \wedge z) \mapsto x \wedge y \wedge z-\zeta \wedge c(x \wedge y \wedge z) /(g-1)
$$

With these definitions, the form $Q_{\wedge^{3} H / H}$ on $\wedge^{3} H / H$ is given by the formula

$$
(u, v) \mapsto(g-1) Q_{\wedge^{3} H}(j(u), j(v))
$$

We denote by $w_{H}, w_{\wedge^{3} H}$, and $w_{\wedge^{3} H / H}$ the $(1,1)$-forms on the intermediate Jacobian fibrations $\mathcal{J}(H), \mathcal{J}\left(\wedge^{3} H\right)$, and $\mathcal{J}\left(\wedge^{3} H / H\right)$ over $\mathcal{A}_{g}$ given by Proposition 4.1. The various morphisms of polarized Hodge structure 
described above canonically give rise to morphisms

$$
\mathcal{J}(H) \stackrel{\wedge \zeta}{\rightleftarrows} \mathcal{c} \mathcal{J}\left(\wedge^{3} H\right) \stackrel{p}{\longrightarrow} \mathcal{J}\left(\wedge^{3} H / H\right)
$$

of intermediate Jacobians over $\mathcal{A}_{g}$.

The key to the proof of Theorem 1.2 is the following.

Proposition 4.3. On $\mathcal{J}\left(\wedge^{3} H\right)$, one has a canonical isometry

$$
\hat{\mathcal{B}}_{\wedge^{3} H}^{\otimes g-1} \stackrel{\sim}{\rightarrow} p^{*} \hat{\mathcal{B}}_{\wedge^{3} H / H} \otimes c^{*} \hat{\mathcal{B}}_{H}
$$

of Hermitian line bundles.

Proof. We have an equality $(g-1) Q_{\wedge^{3} H}=p^{*} Q_{\wedge^{3} H / H}+c^{*} Q_{H}$ of polarization forms on the variation of Hodge structure associated to $\wedge^{3} H$, by [16, Proposition 18]. This implies the equality $(g-1) w_{\wedge^{3} H}=p^{*} w_{\wedge^{3} H / H}+c^{*} w_{H}$ of associated canonical $(1,1)$-forms on $\mathcal{J}\left(\wedge^{3} H\right)$. The Chern forms of the left- and right-hand sides of the claimed isometry are therefore equal by Proposition 4.2. Moreover, both the left- and right-hand sides restrict to the trivial Hermitian line bundle after pulling back along the zero section. By the uniquely defining property from Proposition 4.2, we obtain the existence of the claimed isometry.

Consider once again the intermediate Jacobians $\mathcal{J}^{p}\left(\mathcal{X}_{g} / \mathcal{A}_{g}\right)=\mathcal{J}\left(V_{\mathbb{Z}, 2 p-1}\right)$ for integers $1 \leq p \leq g$ associated to the universal abelian variety $\mathcal{X}_{g} \rightarrow \mathcal{A}_{g}$. Each $\mathcal{J}^{p}\left(\mathcal{X}_{g} / \mathcal{A}_{g}\right)$ admits an Abel-Jacobi map $a_{p}: \mathrm{CH}_{o}^{p}\left(\mathcal{X}_{g} / \mathcal{A}_{g}\right) \rightarrow$ $\mathcal{J}^{p}\left(\mathcal{X}_{g} / \mathcal{A}_{g}\right)$. Let $p, q$ be positive integers such that $p+q=g+1$. Let $\mathrm{CH}_{o}\left(\mathcal{X}_{g} / \mathcal{A}_{g}\right)=\bigoplus_{p=1}^{g} \mathrm{CH}_{o}^{p}\left(\mathcal{X}_{g} / \mathcal{A}_{g}\right)$. By work of Deninger and Murre [11], one has a Fourier-Mukai transform $F$ on the Chow group $\mathrm{CH}^{*}(\mathcal{A} / S)_{\mathbb{Q}}$ of relative cycles with $\mathbb{Q}$-coefficients on each abelian scheme $\mathcal{A} / S$; it respects cohomological triviality. As the construction of the Fourier-Mukai transform commutes with arbitrary base changes, one then has a canonical FourierMukai transform $F: \mathrm{CH}_{o}\left(\mathcal{X}_{g} / \mathcal{A}_{g}\right)_{\mathbb{Q}} \rightarrow \mathrm{CH}_{o}\left(\mathcal{X}_{g} / \mathcal{A}_{g}\right)_{\mathbb{Q}}$, where $\mathcal{X}_{g}$ is identified with its dual $\check{\mathcal{X}}_{g}$ by the tautological principal polarization.

The transform $F$ is compatible with the polarization morphism $i$ : $\mathcal{J}^{p}\left(\mathcal{X}_{g} / \mathcal{A}_{g}\right) \rightarrow \check{\mathcal{J}}^{p}\left(\mathcal{X}_{g} / \mathcal{A}_{g}\right)$ under the Abel-Jacobi maps, in the following sense.

Proposition 4.4. Let $p, q$ be positive integers such that $p+q=g+1$, and let $F_{p q}: \mathrm{CH}_{o}^{p}\left(\mathcal{X}_{g} / \mathcal{A}_{g}\right)_{\mathbb{Q}} \rightarrow \mathrm{CH}_{o}^{q}\left(\mathcal{X}_{g} / \mathcal{A}_{g}\right)_{\mathbb{Q}}$ be the composition of the 
Fourier-Mukai transform $\mathrm{CH}_{o}^{p}\left(\mathcal{X}_{g} / \mathcal{A}_{g}\right)_{\mathbb{Q}} \rightarrow \mathrm{CH}_{o}\left(\mathcal{X}_{g} / \mathcal{A}_{g}\right)_{\mathbb{Q}}$ with the projection map $\mathrm{CH}_{o}\left(\mathcal{X}_{g} / \mathcal{A}_{g}\right)_{\mathbb{Q}} \rightarrow \mathrm{CH}_{o}^{q}\left(\mathcal{X}_{g} / \mathcal{A}_{g}\right)_{\mathbb{Q}}$. Let $i: \mathcal{J}^{p}\left(\mathcal{X}_{g} / \mathcal{A}_{g}\right) \stackrel{\sim}{\rightarrow} \check{\mathcal{J}}^{p}\left(\mathcal{X}_{g} / \mathcal{A}_{g}\right)$ be the polarization isomorphism derived from the tautological polarization on $\mathcal{X}_{g}$. Then $i\left(a_{p}(z)\right)=\operatorname{pd} a_{q}\left(F_{p q}(z)\right)$ for all global sections $z$ of $\mathrm{CH}_{o}^{p}\left(\mathcal{X}_{g} / \mathcal{A}_{g}\right)_{\mathbb{Q}}$.

Proof. In [3, Proposition 2] the analogous result is proved for principally polarized abelian varieties over $\mathbb{C}$. The proposition follows as the equality $i \circ a_{p}=\operatorname{pd} \circ a_{q} \circ F_{p q}$ of maps holds fiber-by-fiber over $\mathcal{A}_{g}$.

\section{$\S 5$. Normal functions associated to the Ceresa cycle}

Let $\mathcal{M}_{g}$ be the moduli space of complex smooth projective curves of genus $g \geq 2$, and let $\pi: \mathcal{M}_{g, 1} \rightarrow \mathcal{M}_{g}$ be the universal curve. Both are viewed as orbifolds. Let $\mathcal{J}(H), \mathcal{J}\left(\wedge^{3} H\right)$, and $\mathcal{J}\left(\wedge^{3} H / H\right)$ be the Griffiths intermediate Jacobian fibrations over $\mathcal{A}_{g}$ determined by the local systems $H, \wedge^{3} H$, and $\wedge^{3} H / H$, respectively, as in Section 4. As explained in [16, Introduction], they fit in a commutative diagram

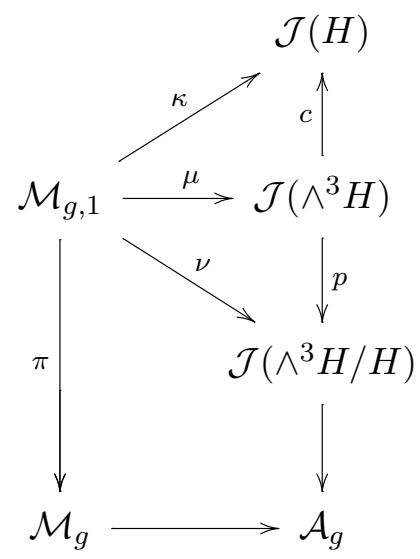

of orbifolds with $p, c$ the maps from Section 4. The normal functions $\kappa, \mu$, and $\nu$ are defined as follows. First of all, $\kappa$ is the map sending a pair $(X, x)$, where $X$ is a curve and $x$ is a point on $X$ to the class of the degree 0 divisor $(2 g-2) x-K$ in the Jacobian $J$ of $X$. Here $K$ is a canonical divisor on $X$.

The map $\mu$ sends a pair $(X, x)$ to the Abel-Jacobi image in $J\left(\wedge^{3} H\right)$ of the Ceresa cycle determined by $x$ and is equal to twice the pointed harmonic volume of $(X, x)$ (see [18], [25]). Recall that the Ceresa cycle is the homologically trivial cycle in the Jacobian $J$ given as $X_{x}-X_{x}^{-}$, where $X_{x}$ is the 
curve $X$ embedded in $J$ via the Abel-Jacobi map $y \mapsto[y-x]$ and where $X_{x}^{-}=[-1]_{*} X_{x}$. The image of $\mu(x)$ under $p$ is independent of the choice of $x$ and in fact we have (see [25, Corollary 6.3]) the relation

$$
\mu(x)-\mu(y)=-2([x-y])
$$

in $J$, sitting canonically inside $J\left(\wedge^{3} H\right)$ by wedging with $\zeta$. We find that the harmonic volume $\nu=p \mu$ factors over $\mathcal{M}_{g}$, and we will denote the resulting normal function $\mathcal{M}_{g} \rightarrow \mathcal{J}\left(\wedge^{3} H / H\right)$ also by $\nu$.

\section{§6. Extension to the Picard variety of degree 1 over $\mathcal{M}_{g}$}

Let $\mathcal{P i c}_{g}^{d} \rightarrow \mathcal{M}_{g}$ be the universal Picard variety in degree $d$, viewed as an orbifold; for example, $\mathcal{P} \mathrm{ic}_{g}^{0}$ is identified with the pullback of $\mathcal{J}(H)$ along the Torelli map $\mathcal{M}_{g} \rightarrow \mathcal{A}_{g}$, that is, the universal Jacobian. The aim of this section is to show that the commutative diagram of Section 5 fits in a larger diagram

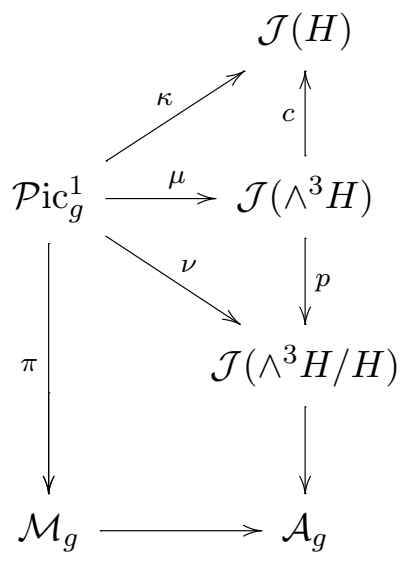

with $\nu$ again factoring over $\mathcal{M}_{g}$. We refer to $[25$, Sections 5,6$]$ for the details of the following explanation. Let $X$ be a compact connected Riemann surface of genus $g \geq 2$, and, as before, let $H=H_{1}(X, \mathbb{Z})$ be its first homology group. We then have the following generalization of the pointed harmonic volume of Section 5. First of all, consider the map

$$
A: J(H) \longrightarrow J\left(\wedge^{3} H\right), \quad[x-y] \mapsto\left[X_{y}-X_{x}\right] .
$$

This map is well defined, and is in fact an injective homomorphism, coinciding with the embedding of $J(H)$ into $J\left(\wedge^{3} H\right)$ given by wedging with $\zeta$ by 
[25, Lemma 6.1]. By [25, Lemma 6.4], we also have a well-defined embedding

$$
S: \operatorname{Pic}^{2} X \longrightarrow J\left(\wedge^{3} H\right), \quad[x+y+D] \mapsto A(D)-\left[X_{x}-X_{y}^{-}\right]
$$

for each degree 0 divisor $D$ on $X$ and $x, y \in X$. It follows that the map

$\mu: \operatorname{Pic}^{1} X \longrightarrow J\left(\wedge^{3} H\right), \quad[x+D] \mapsto-A(2 D)+\left[X_{x}-X_{x}^{-}\right]=-A(2 D)+\mu(x)$

is well defined too. We have a canonical injection $X \rightarrow \operatorname{Pic}^{1} X$, and the map $\mu$ clearly restricts to the pointed harmonic volume on $X$. The image $\mu(e)$ of a point $e$ in $\operatorname{Pic}^{1} X$ represents the class of the cycle $X_{e}-X_{e}^{-}$, where $X_{e}$ is the Abel-Jacobi image of $X$ in $J(H)$ using $e$ as a base divisor.

Proposition 6.1. We have the following.

(a) The map $\mu: \operatorname{Pic}^{1} X \rightarrow J\left(\wedge^{3} H\right)$ is the linear extension of the pointed harmonic volume $\mu: X \rightarrow J\left(\wedge^{3} H\right)$.

(b) We have the equality $\mu(e)-\mu\left(e^{\prime}\right)=-2\left(e-e^{\prime}\right)$ for all $e, e^{\prime}$ in $\operatorname{Pic}^{1} X$. In particular, the image of $\mu(e)$ under $p$ is independent of the choice of $e$.

(c) The set $\mu\left(\operatorname{Pic}^{1} X\right)$ is a coset of $J(H)$ in $J\left(\wedge^{3} H\right)$.

Let $K$ be a canonical divisor on $X$.

(d) The image of $e$ under $c \mu$ equals the class of $(2 g-2) e-K$ in $J(H)$ for all e in $\mathrm{Pic}^{1} X$.

Proof. (a) Take a representative $\sum_{i} m_{i} x_{i}$ of $e$ with $m_{i} \in \mathbb{Z}, x_{i} \in X$, and $\sum_{i} m_{i}=1$. We would like to know whether $\mu(e)=\sum_{i} m_{i} \mu\left(x_{i}\right)$. For each index $i$ we can write

$$
e=\left[x_{i}+\sum_{j} m_{j}^{(i)} x_{j}\right]
$$

where $m_{j}^{(i)}=m_{j}$ if $j \neq i$, and $m_{j}^{(i)}=m_{i}-1$ if $j=i$; note that $\sum_{j} m_{j}^{(i)} x_{j}$ is of degree 0 . It follows that

$$
\mu(e)=-2 A\left(\left[\sum_{j} m_{j}^{(i)} x_{j}\right]\right)+\mu\left(x_{i}\right)
$$

for all $i$. Noting that $\mu(e)=\sum_{i} m_{i} \mu(e)$, we obtain

$$
\mu(e)=\sum_{i} m_{i} \mu(e)=-2 A\left(\left[\sum_{j}\left(\sum_{i} m_{i} m_{j}^{(i)}\right) x_{j}\right]\right)+\sum_{i} m_{i} \mu\left(x_{i}\right) .
$$

But $\sum_{i} m_{i} m_{j}^{(i)}=0$ for all $j$ and the result follows. 
(b) This follows from (a) and equation (5.1) by writing $e=E_{1}-E_{2}$ and $e^{\prime}=E_{1}^{\prime}-E_{2}^{\prime}$ with $E_{1}, E_{2}, E_{1}^{\prime}$, and $E_{2}^{\prime}$ effective divisors, with $\operatorname{deg}\left(E_{1}\right)=$ $\operatorname{deg}\left(E_{1}^{\prime}\right)$.

(c) This follows from (b), noting that the map $\mu: \operatorname{Pic}^{1} X \rightarrow J\left(\wedge^{3} H\right)$ has finite fibers.

(d) This follows from (a) and the fact that $c \mu(x)$ in $J(H)$ is the class of $(2 g-2) x-K$ for each $x$ on $X$, as we saw in Section 5 .

The proposition implies that there exist canonical maps $\mu$ as well as $\kappa=c \mu$ and $\nu=p \mu$ on $\mathcal{P i c}_{g}^{1}$, extending linearly the maps denoted by the same symbol on $\mathcal{M}_{g, 1}$. By linear extension from the original pointed harmonic volume, which is holomorphic, the map $\mu$ is holomorphic on $\mathcal{P i c}_{g}^{1}$.

\section{§7. Proof of Theorem 1.2}

In this section we prove Theorem 1.2. By compatibility with base change Corollary 3.2 it suffices to consider the universal case, where $S$ is the moduli space $\mathcal{P}$ ic $_{g}^{1}$ of pairs $(X, e)$, with $X$ a complex smooth projective curve of genus $g$ and $e$ a point on $\operatorname{Pic}^{1} X$, and where $\pi: \mathcal{X} \rightarrow S$ is the universal curve over $\mathcal{P i c}_{g}^{1}$. Let $C_{e}=X_{e}-X_{e}^{-}$be the flat family of Ceresa cycles on the universal Jacobian $\mathcal{J}$ over $S$. Let $F$ be the Fourier-Mukai transform (see [11]) on the Chow group $\mathrm{CH}^{*}(\mathcal{J} / S)_{\mathbb{Q}}$ of relative cycles of $\mathcal{J} / S$ with $\mathbb{Q}$ coefficients. Given two positive integers $p, q$ with $p+q=g+1$, by abuse of notation we also write $F$ for the composition of the Fourier-Mukai transform $\mathrm{CH}_{o}^{p}\left(\mathcal{X}_{g} / \mathcal{A}_{g}\right)_{\mathbb{Q}} \rightarrow \mathrm{CH}_{o}\left(\mathcal{X}_{g} / \mathcal{A}_{g}\right)_{\mathbb{Q}}$ with the projection map $\mathrm{CH}_{o}\left(\mathcal{X}_{g} / \mathcal{A}_{g}\right)_{\mathbb{Q}} \rightarrow$ $\mathrm{CH}_{o}^{q}\left(\mathcal{X}_{g} / \mathcal{A}_{g}\right)_{\mathbb{Q}}$. In our case, we will have $p=g-1$ and $q=2$.

Proposition 7.1 establishes a connection between $C_{e}$ and the flat family of Gross-Schoen cycles $\Delta_{e}$ on the universal triple product curve $\mathcal{Y}$ over $S$. The proposition generalizes a result on the level of $\mathbb{R}$-valued height pairings from [29, Section 5].

Proposition 7.1. There exists a canonical isometry

$$
\left\langle\Delta_{e}, \Delta_{e}\right\rangle^{\otimes 2} \stackrel{\sim}{\rightarrow}\left\langle C_{e}, F\left(C_{e}\right)\right\rangle^{\otimes 3}
$$

of Hermitian line bundles on $\mathcal{P i c}_{g}^{1}$.

Proof. We have a proper flat map $f: \mathcal{Y} \rightarrow \mathcal{J}$ over $S$ given by sending a tuple $(X, e ; x, y, z)$ to the class of $x+y+z-3 e$ in the Jacobian of $X$. We have $f^{*} F\left(C_{e}\right) \equiv 2 \Delta_{e}$ in $\mathrm{CH}_{o}^{2}(\mathcal{Y} / S)$ by [29, Theorem 1.5.5]. We thus obtain 
a canonical isometry

$$
\left\langle\Delta_{e}, \Delta_{e}\right\rangle^{\otimes 2} \stackrel{\sim}{\rightarrow}\left\langle\Delta_{e}, f^{*} F\left(C_{e}\right)\right\rangle
$$

of Hermitian line bundles on $\mathcal{P i c}_{g}^{1}$. By the projection formula Proposition 2.1(c) and Proposition 2.2, we next have a canonical isometry

$$
\left\langle\Delta_{e}, f^{*} F\left(C_{e}\right)\right\rangle \stackrel{\sim}{\longrightarrow}\left\langle f_{*} \Delta_{e}, F\left(C_{e}\right)\right\rangle .
$$

Finally, we have $f_{*} \Delta_{e} \equiv 3 C_{e}$ in $\mathrm{CH}_{o}^{g-1}(\mathcal{J} / S)$ by [8, Proposition 2.9], yielding a canonical isometry

$$
\left\langle f_{*} \Delta_{e}, F\left(C_{e}\right)\right\rangle \stackrel{\sim}{\rightarrow}\left\langle C_{e}, F\left(C_{e}\right)\right\rangle^{\otimes 3} .
$$

The proposition follows by combining the three isometries.

Let $\kappa, \mu$, and $\nu$ be the normal functions on $\mathcal{P i c}_{g}^{1}$ constructed as a result of the discussion in Section 6.

Proposition 7.2. There exists a canonical isometry

$$
\left\langle C_{e}, F\left(C_{e}\right)\right\rangle \stackrel{\sim}{\rightarrow} \mu^{*} \hat{\mathcal{B}}_{\wedge^{3} H}
$$

of Hermitian line bundles on $\mathcal{P i c}_{g}^{1}$.

Proof. By Proposition 3.3, we have a canonical isometry

$$
\left\langle C_{e}, F\left(C_{e}\right)\right\rangle \stackrel{\sim}{\rightarrow}\left(a\left(C_{e}\right), \operatorname{pd} a\left(F\left(C_{e}\right)\right)\right)^{*} \mathcal{B}_{\wedge^{3} H},
$$

where $\mathcal{B}_{\wedge^{3} H}$ is the Poincaré biextension line bundle on $\mathcal{J}\left(\wedge^{3} H\right) \times \check{\mathcal{J}}\left(\wedge^{3} H\right)$. Here we use $a$ as shorthand for the Abel-Jacobi image. By Proposition 4.4 and by the construction of $\hat{\mathcal{B}}_{\wedge^{3} H}$, we find a chain of equalities

$$
\left(a\left(C_{e}\right), \operatorname{pd} a\left(F\left(C_{e}\right)\right)\right)^{*} \mathcal{B}_{\wedge^{3} H}=\left(a\left(C_{e}\right), i a\left(C_{e}\right)\right)^{*} \mathcal{B}_{\wedge^{3} H}=\mu^{*} \hat{\mathcal{B}}_{\wedge^{3} H} .
$$

We obtain the proposition by combining these two results.

Proposition 7.3. There exists a canonical isometry

$$
\mu^{*} \hat{\mathcal{B}}_{\wedge^{3} H}^{\otimes g-1} \stackrel{\sim}{\rightarrow} \nu^{*} \hat{\mathcal{B}}_{\wedge^{3} H / H} \otimes \kappa^{*} \hat{\mathcal{B}}_{H}
$$

of Hermitian line bundles on $\mathcal{P i c}_{g}^{1}$. 
Proof. By Proposition 4.3, we have a canonical isometry

$$
\hat{\mathcal{B}}_{\wedge^{3} H}^{\otimes g-1} \stackrel{\sim}{\rightarrow} p^{*} \hat{\mathcal{B}}_{\wedge^{3} H / H} \otimes c^{*} \hat{\mathcal{B}}_{H}
$$

of line bundles over $\mathcal{J}\left(\wedge^{3} H\right)$. We obtain the required isometry over $\mathcal{P i c}_{g}^{1}$ by pulling back this isometry along the extended harmonic volume $\mu$, noting that $\kappa=c \mu$ and $\nu=p \mu$.

Proposition 7.4. Let $x_{e}$ be the relative degree 0 divisor class on the universal curve $\mathcal{X}$ over $\mathcal{P i c}_{g}^{1}$ given by $(2 g-2) e-c_{1}(\omega)$, where $\omega$ is the relative dualizing sheaf of $\mathcal{X} / \mathcal{P i c}_{g}^{1}$ and where $e$ is the tautological relative degree 1 divisor. Then there exists a canonical isometry

$$
\kappa^{*} \hat{\mathcal{B}}_{H} \stackrel{\sim}{\rightarrow}\left\langle x_{e}, x_{e}\right\rangle^{\otimes-1}
$$

of Hermitian line bundles on $\mathcal{P i c}_{g}^{1}$.

Proof. By construction, $\hat{\mathcal{B}}_{H}$ is the pullback along (id, $i$ ) of the Poincaré bundle $\mathcal{B}_{H}$ on $\mathcal{J}(H) \times \check{\mathcal{J}}(H)$. We have seen at the end of Section 3 that $\mathrm{pd}=i \circ[-1]$ in this case. Note that the Abel-Jacobi image of $x_{e}$ over $(X, e)$ is equal to $\kappa(X, e)$. We obtain a chain of isometries

$$
\kappa^{*} \hat{\mathcal{B}}_{H} \stackrel{\sim}{\rightarrow}\left(a\left(x_{e}\right), i a\left(x_{e}\right)\right)^{*} \mathcal{B}_{H} \stackrel{\sim}{\rightarrow}\left(a\left(x_{e}\right), \operatorname{pd} a\left(x_{e}\right)\right)^{*} \mathcal{B}_{H}^{\otimes-1} \stackrel{\sim}{\rightarrow}\left\langle x_{e}, x_{e}\right\rangle^{\otimes-1},
$$

with the last isometry given by Proposition 3.3. The proposition follows.

Proof of Theorem 1.2. By combining the above propositions we obtain a canonical isometry of Hermitian line bundles

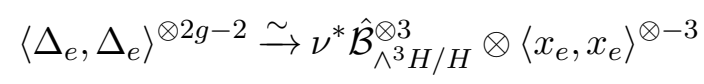

on $\mathcal{P} \mathrm{ic}_{g}^{1}$. The proof of Theorem 1.2 is finished once we show that there exists, up to sign, a canonical isomorphism of line bundles

$$
\left.\nu^{*} \hat{\mathcal{B}}_{\wedge^{3} H / H}^{\otimes 3} \stackrel{\sim}{\rightarrow}\langle\omega, \omega\rangle\right\rangle^{\otimes 2 g+1}
$$

on $\mathcal{M}_{g}$ of norm $\exp (-(2 g-2) \varphi)$, where $\varphi$ is the invariant defined in (1.1). Here $\omega$ is the relative dualizing sheaf of the universal curve over $\mathcal{M}_{g}$, and $\langle\omega, \omega\rangle$ has the metric induced by the Arakelov metric on $\omega$ (see [10]). By a result of Morita (see [16, Theorem 7]), there exists an isomorphism of holomorphic line bundles

$$
\nu^{*} \hat{\mathcal{B}}_{\wedge^{3} H / H} \stackrel{\sim}{\rightarrow}\left(\operatorname{det} \pi_{*} \omega\right)^{\otimes 8 g+4}
$$


on $\mathcal{M}_{g}$. As the only invertible holomorphic functions on $\mathcal{M}_{g}$ are constants (see [17, Lemma 2.1]), the isomorphism is determined up to a scalar.

We can determine this scalar modulo a sign in a canonical way as follows. Consider the hyperelliptic locus $\mathcal{H}_{g}$ inside $\mathcal{M}_{g}$. Then the line bundle $\left(\operatorname{det} \pi_{*} \omega\right)^{\otimes 8 g+4}$ restricted to $\mathcal{H}_{g}$ has (up to sign) a canonical global section $\Lambda_{g}$, given as follows (see [20, Section 2]). Let $U \rightarrow \mathcal{H}_{g}$ be a morphism, with $U$ an integral variety over $\mathbb{C}$, yielding a family of hyperelliptic curves over $U$. Let $y^{2}=f(x)$ with $f(x)=\sum_{i=0}^{2 g+2} a_{i} x^{i}$ and with the $a_{i}$ in the function field of $U$ be a local equation of the family. Then locally over $U$, the section $\Lambda_{g}$ can be written as follows:

$$
\Lambda_{g}=\Delta(f)^{g} \cdot\left(\frac{\mathrm{d} x}{2 y} \wedge \cdots \wedge \frac{x^{g-1} \mathrm{~d} x}{2 y}\right)^{\otimes 8 g+4},
$$

where $\Delta(f)$ is the discriminant of $f$. The section $\Lambda_{g}$ is characterized, up to sign, as a global section of $\left(\operatorname{det} \pi_{*} \omega\right)^{\otimes 8 g+4}$ by the property that its extension as a rational section of $\left(\operatorname{det} \pi_{*} \omega\right)^{\otimes 8 g+4}$ over the moduli stack of hyperelliptic curves of genus $g$ over $\mathbb{Z}$ is regular and nowhere vanishing.

On the other hand, the map $\nu$ restricted to $\mathcal{H}_{g}$ coincides with the zero section (see [17, Proposition 6.7]). It follows that the line bundle $\nu^{*} \hat{\mathcal{B}}_{\wedge^{3} H / H}$ restricted to $\mathcal{H}_{g}$ has a canonical trivialization. The scalar in the Morita isomorphism is therefore determined, up to sign, by letting the canonical trivializing sections of $\nu^{*} \hat{\mathcal{B}}_{\wedge^{3} H / H}$ and of $\left(\operatorname{det} \pi_{*} \omega\right)^{\otimes 8 g+4}$ over $\mathcal{H}_{g}$ correspond.

We next compute the norm of the canonical Morita isomorphism, where $\operatorname{det} \pi_{*} \omega$ has the metric induced from the $L^{2}$-metric on $\pi_{*} \omega$ given by $\|\alpha\|^{2}=$ $\frac{i}{2} \int \alpha \wedge \bar{\alpha}$. Let $\delta_{F}$ be Faltings's delta-invariant (see [12]) on $\mathcal{M}_{g}$, and put $\delta=-4 g \log (2 \pi)+\delta_{F}$. Let $\lambda$ be Zhang's lambda-invariant in [29, Section 1.4], determined by the equality

$$
\lambda=\frac{g-1}{6(2 g+1)} \varphi+\frac{1}{12} \delta .
$$

Then the main result of [9] implies that the norm of the canonical Morita isomorphism is equal to $\exp (-(8 g+4) \lambda)$.

Note that there is, up to sign, a canonical isomorphism $\left(\operatorname{det} \pi_{*} \omega\right)^{\otimes 12} \stackrel{\sim}{\rightarrow}$ $\langle\omega, \omega\rangle$ of line bundles on $\mathcal{M}_{g}$ due to Mumford. According to Faltings [12, Theorem 6] and Moret-Bailly [23, Théorème 2.2], the norm of the Mumford isomorphism equals $\exp (\delta)$, if $\langle\omega, \omega\rangle$ is endowed with the metric induced from the Arakelov metric on $\omega$. Combining the Mumford and Morita isomorphisms we obtain, up to sign, a canonical isomorphism $\nu^{*} \hat{\mathcal{B}}_{\wedge^{3} H / H}^{\otimes 3} \stackrel{\sim}{\rightarrow}$ $\langle\omega, \omega\rangle^{\otimes 2 g+1}$ which has norm $\exp (-(2 g-2) \varphi)$, as required. 
It would be interesting to have a way of determining the Morita isomorphism (and hence the isomorphism in Theorem 1.2) that does not refer to the locus of hyperelliptic curves.

The next result was announced in the introduction.

Proposition 7.5. Let $S$ be a smooth quasiprojective variety over the complex numbers. Let $\pi: \mathcal{X} \rightarrow S$ be a smooth projective family of curves of genus $g \geq 2$ over $S$. Then the function $t \mapsto \varphi\left(\mathcal{X}_{t}\right)$ is a continuous function on $S$.

Proof. Let $G: \mathcal{X} \times_{S} \mathcal{X} \rightarrow \mathbb{R}_{\geq 0}$ be the Arakelov-Green's function (see [1], [12]) on the fiber product $\mathcal{X} \times{ }_{S} \mathcal{X}$. This puts a canonical $C^{\infty}$-Hermitian metric on the line bundle $\mathcal{O}(\Delta)$ on $\mathcal{X} \times{ }_{S} \mathcal{X}$, where $\Delta$ is the diagonal. Let $\eta$ be the curvature $(1,1)$-form of $\mathcal{O}(\Delta)$ with respect to this metric. Proposition 2.5.3 of [29] shows that

$$
\varphi\left(\mathcal{X}_{t}\right)=\int_{\mathcal{X}_{t}} \log G \cdot \eta^{2} .
$$

The continuity of the fiber integral follows from [7, Proposition 1.5.1].

\section{§8. Extension to stable curves of compact type}

Let $\mathcal{M}_{g}^{c}$ denote the orbifold of stable curves of genus $g \geq 2$ of compact type, that is, of stable curves such that the associated Jacobian has trivial toric part. Let $\mathcal{P i c}_{g}^{1, c} \rightarrow \mathcal{M}_{g}^{c}$ denote the universal degree 1 part of the Picard variety over $\mathcal{M}_{g}^{c}$. The objective of this section is to extend the Hermitian line bundle $\left\langle\Delta_{e}, \Delta_{e}\right\rangle$ over $\mathcal{P i c}_{g}^{1, c}$, and to prove Theorems 1.3 and 1.4. A basic reference for this section is [15] (esp. Section 5).

First of all, note that by pulling back the various variations of polarized Hodge structure on $\mathcal{A}_{g}$ constructed in Section 4 along the Torelli map $\mathcal{M}_{g}^{c} \rightarrow \mathcal{A}_{g}$ we obtain variations of polarized Hodge structure over $\mathcal{M}_{g}^{c}$, together with their associated intermediate Jacobian fibrations $\mathcal{J}\left(V_{\mathbb{Z}}\right)$ endowed with a canonical metrized biextension line bundle and canonical invariant $(1,1)$-form $w_{V}$. By the discussion in [15, Section 5], and linear extension, each of the normal functions $\kappa, \mu, \nu$ defined above on $\mathcal{P i c}_{g}^{1}$ extends as a normal function into the appropriate intermediate Jacobian fibration over $\mathcal{M}_{g}^{c}$. In particular, we have a canonical Hermitian line bundle $\mu^{*}\left(\hat{\mathcal{B}}_{\wedge^{3} H}\right)$ over $\mathcal{P} \mathrm{ic}_{g}^{1, c}$. It is the unique extension of the Hermitian line bundle $\mu^{*}\left(\hat{\mathcal{B}}_{\wedge^{3} H}\right)$ over $\mathcal{P} \mathrm{ic}_{g}^{1}$ as a Hermitian line bundle over $\mathcal{P i c}_{g}^{1, c}$. 
By Propositions 7.1 and 7.2 above, we have a canonical isometry $\left\langle\Delta_{e}, \Delta_{e}\right\rangle^{\otimes 2} \stackrel{\sim}{\rightarrow} \mu^{*}\left(\hat{\mathcal{B}}_{\wedge^{3} H}^{\otimes 3}\right)$ over $\mathcal{P i c}_{g}^{1}$. We conclude that there exists a unique extension of the Bloch pairing $\left\langle\Delta_{e}, \Delta_{e}\right\rangle^{\otimes 2}$ as a Hermitian line bundle over $\mathcal{P i c}_{g}^{1, c}$. In particular, we have a canonical extension of $\left\langle\Delta_{e}, \Delta_{e}\right\rangle^{\otimes 2}$ as a line bundle over $\mathcal{P} \mathrm{ic}_{g}^{1, c}$. Almost by construction, the following proposition holds.

Proposition 8.1. We have a canonical isometry $\left\langle\Delta_{e}, \Delta_{e}\right\rangle^{\otimes 2} \stackrel{\sim}{\rightarrow} \mu^{*}\left(\hat{\mathcal{B}}_{\wedge^{3} H}^{\otimes 3}\right)$ of Hermitian line bundles over $\mathcal{P i c}_{g}^{1, c}$.

Theorem 1.3 is obtained by combining Proposition 8.1 with the following general result.

THEOREM 8.2. Let $M$ be a complex manifold, and let $\mathcal{V}$ be a variation of polarized integral Hodge structures of weight -1 on $M$. Let $\mathcal{J}(V) \rightarrow M$ be the associated intermediate Jacobian fibration, let $\mu: M \rightarrow \mathcal{J}(V)$ be a normal function, and let $w_{V}$ be the canonical invariant $(1,1)$-form on $\mathcal{J}(V)$. Then $\mu^{*} w_{V}$ is a nonnegative $(1,1)$-form on $M$.

Proof. For the proof, see [15, Theorem 13.1].

Proof of Theorem 1.3. From Proposition 8.1 it follows that the Chern form of $\left\langle\Delta_{e}, \Delta_{e}\right\rangle^{\otimes 2}$ and the Chern form of $\mu^{*}\left(\hat{\mathcal{B}}_{\wedge^{3} H}^{\otimes 3}\right)$ are equal on $\mathcal{P i c}_{g}^{1, c}$. By Proposition 4.2 , the Chern form of $\mu^{*}\left(\hat{\mathcal{B}}_{\wedge^{3} H}\right)$ is equal to $2 \mu^{*} w_{\wedge^{3} H}$, where $w_{\wedge^{3} H}$ is the canonical invariant $(1,1)$-form on $\mathcal{J}\left(\wedge^{3} H\right)$. Theorem 8.2 then implies that the Chern form of $\mu^{*}\left(\hat{\mathcal{B}}_{\wedge^{3} H}\right)$ is nonnegative.

Our proof of Theorem 1.4 is based on class calculations by Hain and Reed [17] and Hain [15].

Proof of Theorem 1.4. We assume from now on that $g \geq 3$, so that $\operatorname{Pic}\left(\mathcal{M}_{g, 1}^{c}\right)$ is torsion-free. Our starting point is the canonical isomorphism

$$
\hat{\mathcal{B}}_{\wedge^{3} H}^{\otimes g-1} \stackrel{\sim}{\rightarrow} p^{*} \hat{\mathcal{B}}_{\wedge^{3} H / H} \otimes c^{*} \hat{\mathcal{B}}_{H}
$$

of line bundles on the intermediate Jacobian fibration $\mathcal{J}\left(\wedge^{3} H\right)$ over $\mathcal{A}_{g}$, provided by Proposition 4.3. By pulling back along the map $\mu: \mathcal{M}_{g, 1}^{c} \rightarrow$ $\mathcal{J}\left(\wedge^{3} H\right)$, we obtain, analogously to Proposition 7.3 , a canonical isomorphism

$$
\mu^{*} \hat{\mathcal{B}}_{\wedge^{3} H}^{\otimes g-1} \stackrel{\sim}{\rightarrow} \nu^{*} \hat{\mathcal{B}}_{\wedge^{3} H / H} \otimes \kappa^{*} \hat{\mathcal{B}}_{H}
$$


of line bundles on $\mathcal{M}_{g, 1}^{c}$. By [17, Theorem 1.3] we have the equality

$$
\nu^{*} \hat{\mathcal{B}}_{\wedge^{3} H / H}=(8 g+4) \lambda-\sum_{i=1}^{g-1} 4 i(g-i) \delta_{i}^{\{x\}}
$$

in $\operatorname{Pic}\left(\mathcal{M}_{g, 1}^{c}\right)$, and by $[15$, Theorem 10.2] we have

$$
\kappa^{*} \hat{\mathcal{B}}_{H}=4 g(g-1) \psi-12 \lambda-\sum_{i=1}^{g-1} 4 i(i-1) \delta_{g-i}^{\{x\}} .
$$

By combining, we obtain

$$
\mu^{*} \hat{\mathcal{B}}_{\wedge^{3} H}=4 g \psi+8 \lambda-\sum_{i=1}^{g-1} 4 i \delta_{g-i}^{\{x\}} .
$$

Using Proposition 8.1, we obtain the required expression for $\left\langle\Delta_{e}, \Delta_{e}\right\rangle$.

\section{REFERENCES}

[1] S. Y. Arakelov, An intersection theory for divisors on an arithmetic surface (in Russian), Izv. Akad. Nauk. SSSR Ser. Mat. 38 (1974), 1179-1192. MR 0472815.

[2] E. Arbarello and M. Cornalba, The Picard groups of the moduli spaces of curves, Topology 26 (1987), 153-171. MR 0895568. DOI 10.1016/0040-9383(87)90056-5.

[3] A. Beauville, "Quelques remarques sur la transformation de Fourier dans l'anneau de Chow d'une variété abelienne" in Algebraic Geometry (Tokyo/Kyoto, 1982), Lecture Notes in Math. 1016, Springer, Berlin, 1983, 238-260. MR 0726428. DOI 10.1007/ BFb0099965.

[4] A. Beilinson, "Height pairing between algebraic cycles" in Current Trends in Arithmetical Algebraic Geometry (Arcata, Calif., 1985), Contemp. Math. 67, Amer. Math. Soc., Providence, 1987, 1-24. MR 0902590. DOI 10.1090/conm/067/902590.

[5] S. Bloch, "Height pairings for algebraic cycles" in Proceedings of the Luminy Conference on Algebraic K-theory (Luminy, 1983), J. Pure Appl. Algebra 34, 1984, 119-145. MR 0772054. DOI 10.1016/0022-4049(84)90032-X.

[6] - "Cycles and biextensions" in Algebraic K-theory and Algebraic Number Theory (Honolulu, 1987), Contemp. Math. 83, Amer. Math. Soc., Providence, 1989, 19-30. MR 0991974. DOI 10.1090/conm/083/991974.

[7] J.-B. Bost, H. Gillet, and C. Soulé, Heights of projective varieties and positive Green forms, J. Amer. Math. Soc. 7 (1994), 903-1027. MR 1260106. DOI 10.2307/2152736.

[8] E. Colombo and B. van Geemen, Note on curves in a Jacobian, Compos. Math. 88 (1993), 333-353. MR 1241954.

[9] R. de Jong, Second variation of Zhang's $\lambda$-invariant on the moduli space of curves, Amer. J. Math. 135 (2013), 275-290. MR 3022965. DOI 10.1353/ajm.2013.0008.

[10] P. Deligne, "Le déterminant de la cohomologie" in Current Trends in Arithmetical Algebraic Geometry (Arcata, Calif., 1985), Contemp. Math. 67, Amer. Math. Soc., Providence, 1987, 93-177. MR 0902592. DOI 10.1090/conm/067/902592. 
[11] C. Deninger and J. Murre, Motivic decomposition of abelian schemes and the Fourier transform, J. Reine Angew. Math. 422 (1991), 201-219. MR 1133323.

[12] G. Faltings, Calculus on arithmetic surfaces, Ann. of Math. (2) 119 (1984), 387-424. MR 0740897. DOI 10.2307/2007043.

[13] B. H. Gross and C. Schoen, The modified diagonal cycle on the triple product of a pointed curve, Ann. Inst. Fourier (Grenoble) 45 (1995), 649-679. MR 1340948.

[14] R. Hain, Biextensions and heights associated to curves of odd genus, Duke Math. J. 61 (1990), 859-898. MR 1084463. DOI 10.1215/S0012-7094-90-06133-2.

[15] - "Normal functions and the geometry of moduli spaces of curves" in Handbook of Moduli, Adv. Lectures Math. 24, International Press, Boston, 2013, 527-556.

[16] R. Hain and D. Reed, Geometric proofs of some results of Morita, J. Algebraic Geom. 10 (2001), 199-217. MR 1811554.

[17] - On the Arakelov geometry of moduli spaces of curves, J. Differential Geom. 67 (2004), 195-228. MR 2153077.

[18] B. Harris, Harmonic volumes, Acta Math. 150 (1983), 91-123. MR 0697609. DOI 10. 1007/BF02392968.

[19] B. Iversen, Cohomology of Sheaves, Universitext, Springer, Berlin, 1986. MR 0842190. DOI 10.1007/978-3-642-82783-9.

[20] I. Kausz, A discriminant and an upper bound for $\omega^{2}$ for hyperelliptic arithmetic surfaces, Compos. Math. 115 (1999), 37-69. MR 1671741. DOI 10.1023/A: 1000580901251.

[21] O. Meyer, Über Biextensionen und Höhenpaarungen algebraischer Zykel, Ph.D. dissertation, University of Regensburg, Regensburg, Germany, 2003.

[22] L. Moret-Bailly, "Métriques permises" in Seminar in Arithmetic Bundles: The Mordell Conjecture (Paris, 1983/84), Astérisque 127, Soc. Math. France, Paris, 1985, 29-87. MR 0801918.

[23] — La formule de Noether pour les surfaces arithmétiques, Invent. Math. 98 (1989), 491-498. MR 1022303. DOI 10.1007/BF01393833.

[24] S. Müller-Stach, $\mathbb{C}^{*}$-extensions of tori, higher Chow groups and applications to incidence equivalence relations for algebraic cycles, K-Theory 9 (1995), 395-406. MR 1351945. DOI 10.1007/BF00961471.

[25] M. J. Pulte, The fundamental group of a Riemann surface: Mixed Hodge structures and algebraic cycles, Duke Math. J. 57 (1988), 721-760. MR 0975119. DOI 10.1215/ S0012-7094-88-05732-8.

[26] M. Seibold, Bierweiterungen für algebraische Zykel und Poincarébundel, Ph.D. dissertation, University of Regensburg, Regensburg, Germany, 2007.

[27] T. Szamuely, Galois Groups and Fundamental Groups, Cambridge Stud. Adv. Math. 117, Cambridge University Press, Cambridge, 2009. MR 2548205. DOI 10.1017/ CBO9780511627064.

[28] S.-W. Zhang, Admissible pairing on a curve, Invent. Math. 112 (1993), 171-193. MR 1207481. DOI 10.1007/BF01232429.

[29] - Gross-Schoen cycles and dualising sheaves, Invent. Math. 179 (2010), 1-73. MR 2563759. DOI 10.1007/s00222-009-0209-3.

[30] - Positivity of heights of codimension 2 cycles over function field of characteristic 0, preprint, arXiv:1001.4788v1 [math.AG]. 
Mathematical Institute

University of Leiden

$2300 R A$

Leiden

The Netherlands

rdejong@math.leidenuniv.nl 